\section{The performance of the Xpert MTB/ RIF Version G4 in a low tuberculosis incidence setting}

Sir,

Mycobacterial testing continues to evolve with improving technology, affecting the way we diagnose, report and interpret mycobacterial results. Prior to introduction of the GeneXpert Ultra, our Mycobacterial Reference Laboratory (MRL) at the Victorian Infectious Diseases Reference Laboratory (VIDRL) in Melbourne, utilised the Xpert MTB/RIF assay, Version G4 (Cepheid, USA), to detect Mycobacterium tuberculosis complex (MTBC) and potential resistance to rifampicin via mutations within a region of the $r p o \mathrm{~B}$ gene. ${ }^{1}$

Prior literature on the performance of this Xpert MTB/RIF assay relates to previous versions of the assay. ${ }^{1}$ Here, we sought to characterise the performance of the G4 Xpert MTB/ RIF for detection of MTBC and identification of potential rifampicin resistance in a low TB prevalence population.

Retrospective data at the state MRL were analysed from 21 June 2012 to 14 June 2018. Processing of primary specimens for acid fast bacilli (AFB) staining, predominantly by the Ziehl-Neelsen method, and mycobacterial culture were routinely performed at the referring laboratory. All mycobacterial culture positive samples were requested to be referred for further characterisation.

Respiratory samples were processed in line with recommendations., ${ }^{2,3}$ Fluids and homogenised extra-pulmonary tissue samples were processed using an accredited in-house method. For paraffin embedded tissue, samples were dewaxed and lysed with a Tris- $\mathrm{HCl}$ digestion buffer prior to testing. The $543 \mathrm{bp}$ region of the $r p o \mathrm{~B}$ gene was sequenced using the primers and methods described by Campbell et al. ${ }^{4}$ on isolates that had an $r p o \mathrm{~B}$ mutation detected via Xpert MTB/RIF assay. Sequences were aligned with the $\mathrm{H}_{37} \mathrm{Rv}$ wild-type sequence to assess for variance.

Phenotypic susceptibility testing was performed on MTBC isolates grown from the same or contemporaneous patient samples using the BD BACTEC MGIT 960 module (Becton Dickinson Microbiology Systems, USA). Phenotypic rifampicin susceptibility was tested at a critical concentration of $1 \mu \mathrm{g} /$ $\mathrm{mL} .^{5}$ Isolates found to have a single nucleotide polymorphism (SNP) that were not known to be associated with rifampicin phenotypic resistance were re-tested using lower concentrations of rifampicin $(0.125 \mu \mathrm{g} / \mathrm{mL}, 0.25 \mu \mathrm{g} / \mathrm{mL}, 0.5 \mu \mathrm{g} / \mathrm{mL})$ to determine if low level phenotypic resistance was present.

Data from all Xpert MTB/RIF tests performed at the MRL were extracted, including AFB results, culture results and, where performed, MTBC rifampicin phenotypic susceptibility and rpoB gene sequencing. Additional data included patient demographics and clinical details such as previous treatment and susceptibility results. Multiple samples from individual patients were included.

Over the study period, 1849 Xpert MTB/RIF tests were performed, of which 681 samples $(36.8 \%)$ were Xpert MTB/ RIF positive (Fig. 1A). This rate decreased to $29.2 \%$ when only primary clinical samples were included. Of the primary samples, $48.7 \%$ were non-respiratory.

A corresponding positive MTBC culture was identified for $47.3 \%$ (460/972) primary samples tested by Xpert MTB/RIF (Table 1). Of the 460 MTBC culture positive primary samples, 85\% (390/460) were positive. In addition, one sample positive by Xpert MTB/RIF and confirmed by second assay ${ }^{6}$ was MTBC culture negative, attributed to overgrowth by Mycobacterium avium complex. A further 4.2\% (18/430) demonstrated discordant Xpert MTB/RIF assay positive, culture negative results. However, 12 of these 18 positive samples were from patients who either had a history of prior or current treatment. The overall sensitivity was $84.8 \%$ [390/ $460,95 \%$ confidence interval (CI) 81.2-87.9\%] and specificity $96.3 \%$ (19/493, 95\% CI 94.3-97.8\%). The Xpert MTB/RIF had a high negative predictive value of $87.6 \%$ (95\% CI 85.0-89.7\%) when compared to culture.

The Xpert MTB/RIF performance was assessed on AFB positive and negative samples. AFB results were unavailable for 15 samples (Table $2 \mathrm{~A}$ ). The positive predictive values for both AFB positive and AFB negative samples were high, 99.4\% (95\% CI 96.1-100\%) and 98.6\% (95\% CI 95.7$99.5 \%$ ) respectively (Table $2 \mathrm{~B}$ ). This reflects a combination of a highly specific assay, coupled with low rates of re-testing of current or previously treated patients. Similarly, the negative predictive value for AFB positive samples was high, 97.5\% (95\% CI 84.7-99.6\%), representing a higher potential burden of target. In samples which were AFB negative, the negative predictive value decreased to $86.3 \%$ (95\% CI 83.6$88.6 \%$ ) but remained high. AFB negative samples from a non-respiratory source had a lower detection rate than respiratory samples $(70.7 \%$ vs $84.3 \%$ ), likely representing a relatively low bacterial sample load, less efficient DNA extraction or a combination of both.

Other authors have previously described Xpert MTB/RIF assay positive, MTBC culture negative results as false positive results, attributed to the assay's suboptimal specificity. ${ }^{8}$ The Xpert MTB/RIF is designed to detect DNA, not viability. Treated patients may not have cleared the non-viable MTB at the time of testing. ${ }^{8}$ This factor needs to be considered when interpreting positive results in this cohort. This issue is expected to be magnified with the introduction of the more sensitive Xpert MTB/RIF Ultra ${ }^{8}$ which utilises multi-copy targets, in addition to the $r p o \mathrm{~B}$ gene, to improve the limit of detection.

A further benefit of the Xpert MTB/RIF assay is the ability to detect potential rifampicin resistance. In this study, the positive and negative predictive values for resistance detection were $93.6 \%$ (95\% CI 78.5-98.3\%) and $100 \%$. A resistance mutation was detected in $4.6 \%$ of samples $(31 / 681$ positive results, from 29 unique patients). There was insufficient signal to determine presence of a mutation in six samples; two were phenotypically rifampicin susceptible, and four had no corresponding culture. There was no Xpert MTB/ RIF undetected phenotypic resistant isolates. Given the numbers in our study, this is not surprising. The Xpert MTB/ RIF assay targets the $81 \mathrm{bp}$ region of the $r p o \mathrm{~B}$ gene where $95-97 \%$ of resistance-conferring mutations are located. ${ }^{9}$ The Ser531Leu amino acid change was the most frequently observed SNP, seen in 16 of our samples, consistent with published reports ${ }^{10}$ (see Fig. 1B). Other SNP changes detected that displayed phenotypic resistance and reduced rifampicin susceptibility have also been described in other studies. ${ }^{10}$ One sample was found to have Leu533Pro, a SNP described as conferring low level rifampicin resistance, ${ }^{11}$ that tested phenotypically resistant in our laboratory; however, this would not have been reliably detected on previous versions of the assay. The clinical significance of low level rifampicin resistance is unresolved; however, patients 


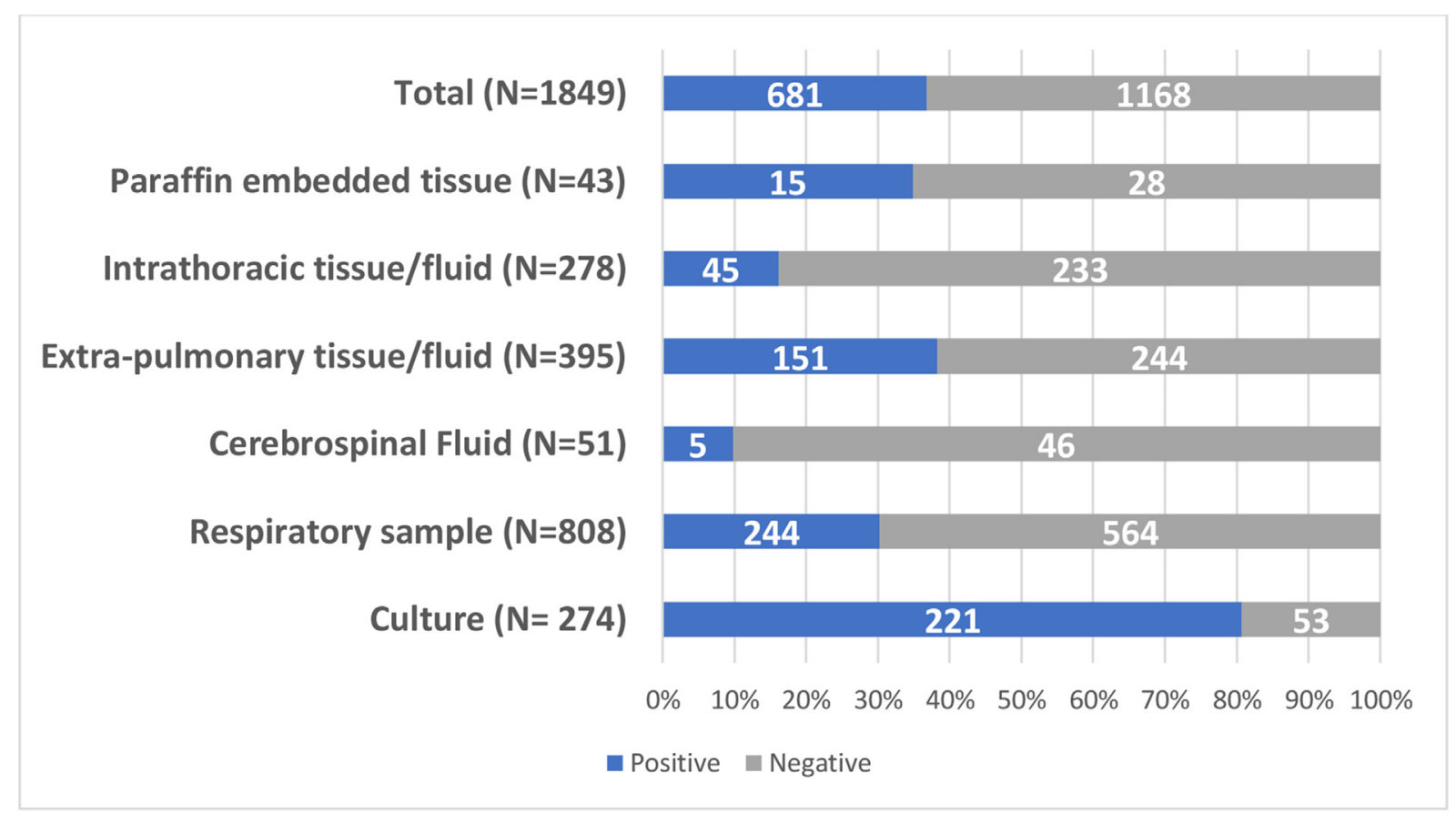

A



B

Fig. 1 (A) Xpert MTB/RIF result by sample type tested from samples received between 21 June 2012 and 14 June 2018 at the Mycobacterial Reference Laboratory. (B) Phenotypic susceptibility mapped against the rpoB sequencing results of Xpert MTB/RIF positive patients with amino acid substitutions detected when aligned with the $\mathrm{H}_{37} \mathrm{Rv}$ wild-type sequence. $\mathrm{R}$, resistance; RS, reduced susceptibility; S, substitution but susceptible.

infected with such isolates often fail treatment or relapse. ${ }^{10}$ Importantly, the MGIT broth susceptibility testing doesn't reliably detect low level phenotypic resistance. ${ }^{10}$ The Xpert MTB/RIF permits identification of these isolates and may allow altered management, including closer monitoring of these at risk patients.

Two Xpert MTB/RIF positive resistance results were phenotypically susceptible. One result was subsequently identified as a true false positive resistance detection. This reflects a potential issue with either the sample preparation, test process or result interpretation on initial testing. The second result had a SNP detected with potential resistance association but tested phenotypically susceptible. Of note, the same mutation was detected in a different isolate that was phenotypically resistant to rifampicin. This highlights that the significance of many SNP changes have yet to be fully elucidated within 
Table 1 Xpert MTB/RIF concordance with culture result

\begin{tabular}{ll}
\hline Culture result & $\begin{array}{c}\text { Positive/Number tested } \\
(\% \text { positive) }\end{array}$ \\
\hline No culture & $51 / 603(8.5 \%)$ \\
Culture negative & $18^{\mathrm{a}} / 430(4.2 \%)$ \\
Non-tuberculous & $1^{\mathrm{b}} / 82(1.2 \%)$ \\
mycobacteria/Nocardia & $390 / 460(84.6 \%)$ \\
\hline
\end{tabular}

a $12 / 18$ noted to have a prior history of treatment or treated at time of collection.

${ }^{\mathrm{b}}$ One sample overgrown with Mycobacterium avium complex.

Table 2 (A) Xpert MTB/RIF result by MTBC culture positive separated by AFB stain result and sample type; (B) Comparison of MTBC culture and Xpert MTB/RIF results by AFB stain result of samples received at the MRL

\begin{tabular}{|c|c|c|c|c|c|}
\hline \multicolumn{6}{|c|}{ (A) Xpert MTB/RIF result by positive MTBC culture } \\
\hline \multirow{2}{*}{\multicolumn{2}{|c|}{ Sample type }} & \multirow{2}{*}{\multicolumn{2}{|c|}{$\begin{array}{c}\text { AFB positive } \\
\begin{array}{c}\text { Positive/Number } \\
\text { tested }\end{array} \\
(\% \text { positive })\end{array}$}} & \multirow{2}{*}{\multicolumn{2}{|c|}{$\begin{array}{c}\text { AFB negative } \\
\begin{array}{c}\text { Positive/Number } \\
\text { tested }\end{array} \\
\text { (\% positive })\end{array}$}} \\
\hline & & & & & \\
\hline \multirow{6}{*}{\multicolumn{2}{|c|}{$\begin{array}{l}\text { Respiratory } \\
\text { Cerebrospinal fluid (CSF) } \\
\text { Extra-pulmonary tissue/fluid } \\
\text { Intrathoracic tissue/fluid sample } \\
\text { Paraffin embedded tissue } \\
\text { All samples }\end{array}$}} & \multirow{6}{*}{\multicolumn{2}{|c|}{$\begin{array}{l}137 / 138(99.3 \%) \\
1 / 1(100 \%) \\
27 / 27(100 \%) \\
7 / 7(100 \%) \\
0 \\
172 / 173(99.4 \%)\end{array}$}} & \multirow{6}{*}{\multicolumn{2}{|c|}{$\begin{array}{l}86 / 102(84.3 \%) \\
4 / 6(66.7 \%) \\
82 / 108(75.9 \%) \\
30 / 50(60.0 \%) \\
5 / 6(83.3 \%) \\
207 / 272(76.1 \%)\end{array}$}} \\
\hline & & & & & \\
\hline & & & & & \\
\hline & & & & & \\
\hline & & & & & \\
\hline & & & & & \\
\hline \multicolumn{6}{|c|}{$\begin{array}{l}\text { (B) Comparison of MTBC culture and Xpert MTB/RIF by AFB stain } \\
\text { result }\end{array}$} \\
\hline & \multicolumn{2}{|c|}{ AFB positive } & \multicolumn{3}{|c|}{ AFB negative } \\
\hline & $\begin{array}{l}\text { Culture } \\
\text { positive }\end{array}$ & $\begin{array}{l}\text { Culture } \\
\text { negative }\end{array}$ & & & $\begin{array}{l}\text { Culture } \\
\text { negative }\end{array}$ \\
\hline Xpert positive & 172 & 1 & 207 & & 3 \\
\hline Xpert negative & 1 & 39 & 65 & & 410 \\
\hline
\end{tabular}

MTBC, and that the Xpert MTB/RIF should be used as a guide to determine potential presence of resistance.

In this retrospective data analysis, the Xpert MTB/RIF assay demonstrated high positive and negative predictive values in both AFB positive and negative samples. Since conducting this analysis, the GeneXpert Ultra has been introduced which includes multi-copy targets, further improving the limit of MTBC detection. The impact on specificity of this change needs to be established. The Xpert MTB/RIF assay has demonstrated its reliability and robustness as the cornerstone of rapid MTBC diagnostics. This report provides a clear benchmark with which to compare newer generation assays.

Conflicts of interest and sources of funding: The authors state that there are no conflicts of interest to disclose.

David A. Foley ${ }^{1,2}$, Linny K. Phuong ${ }^{3,4}$, Maria Globan ${ }^{1}$, Janet M. Fyfe ${ }^{1}$, Caroline Lavender ${ }^{1}$, Deborah A. Williamson ${ }^{1,5,6}$

${ }^{1}$ Mycobacterial Reference Laboratory, Victorian Infectious Diseases Reference Laboratory, Melbourne, Vic, Australia;
${ }^{2}$ Department of Infectious Diseases, Perth Children's Hospital, Nedlands, WA, Australia; ${ }^{3}$ Department of General Medicine, Infectious Diseases Unit, Royal Children's Hospital, Parkville, Vic, Australia; ${ }^{4}$ Murdoch Children's Research Institute, Parkville, Vic, Australia; ${ }^{5}$ Department of Microbiology, Royal Melbourne Hospital, Parkville, Vic, Australia; ${ }^{6}$ Microbiological Diagnostic Unit Public Health Laboratory, Peter Doherty Institute, Melbourne, Vic, Australia

Contact Dr David Foley.

E-mail: drdavidanthonyfoley@gmail.com

1. Nicol MP, Whitelaw A, Wendy S. Using Xpert MTB/RIF. Curr Respir Med Rev 2013; 9: 187-92.

2. World Health Organization (WHO). Xpert MTB/RIF Implementation Manual. Technical and Operational 'How-To': Practical Considerations. Geneva: WHO, 2014.

3. Lawn SD, Nicol MP. Xpert® MTB/RIF assay: development, evaluation and implementation of a new rapid molecular diagnostic for tuberculosis and rifampicin resistance. Future Microbiol 2011; 6: 1067-82.

4. Campbell PJ, Morlock GP, Sikes RD, et al. Molecular detection of mutations associated with first- and second-line drug resistance compared with conventional drug susceptibility testing of Mycobacterium tuberculosis. Antimicrob Agents Chemother 2011; 55: 2032-41.

5. Woods GL, Lin S-YG, Desmond EP. Susceptibility test methods: mycobacteria, nocardia, and other actinomycetes. In: Versalovic J, Carroll KC, Funke G, et al., editors. Manual of Clinical Microbiology. 10th ed. Washington, DC: ASM Press, 2011; 1215-38.

6. Globan M, Fyfe J. Mycobacterium tuberculosis complex. In: Schuller M, Sloots T, James G, Halliday C, Carter I, editors. PCR for Clinical Microbiology. Dordrecht: Springer, 2010.

7. Scott LE, Beylis N, Nicol M, et al. Diagnostic accuracy of Xpert MTB/ RIF for extrapulmonary tuberculosis specimens: establishing a laboratory testing algorithm for South Africa. J Clin Microbiol 2014; 52: $1818-23$.

8. Theron G, Venter R, Smith L, et al. False-positive Xpert MTB/RIF results in retested patients with previous tuberculosis: frequency, profile, and prospective clinical outcomes. J Clin Microbiol 2018; 56 e01696-17.

9. Ramaswamy S, Musser JM. Molecular genetic basis of antimicrobial agent resistance inMycobacterium tuberculosis: 1998 update. Tubercle Lung Dis 1998; 79: 3-29.

10. Al-Mutairi NM, Ahmad S, Mokaddas E, et al. Occurrence of disputed rpoB mutations among Mycobacterium tuberculosis isolates phenotypically susceptible to rifampicin in a country with a low incidence of multidrug-resistant tuberculosis. BMC Infect Dis 2019; 19: 3.

11. Somoskovi A, Deggim V, Ciardo D, et al. Diagnostic implications of inconsistent results obtained with the Xpert MTB/Rif assay in detection of Mycobacterium tuberculosis isolates with an rpoB mutation associated with low-level rifampin resistance. J Clin Microbiol 2013; 51 $3127-9$.

DOI: https://doi.org/10.1016/j.pathol.2021.03.007

\section{Ignavigranum ruoffiae associated with skin abscess}

Sir,

We describe the isolation of Ignavigranum ruoffiae from a skin abscess in a patient attending our emergency department. A 62-year-old female patient presented to hospital with an infected sebaceous cyst and overlying cellulitis. She underwent operative debridement with tissue/sebaceous cyst collected for histopathology and pus sent for microbiological analysis. Gram-positive cocci were seen on microscopy and the patient was discharged on oral flucloxacillin with good clinical response. Pure growth of an unusual pathogen, 